\title{
ENVIRONMENTAL CONTAMINATION BY SUBSTRATA OF ORE MINING DUMPS, THEIR MONITORING AS WELL AS MEASURES OF REDUCTION
}

\author{
Blumenstein O. ${ }^{1}$, Pustlauck F. ${ }^{1}$ and Vavelidis M. ${ }^{2}$ \\ ${ }^{1}$ University of Potsdam, Institute of Earth and Environmental Science, Research Group "Applied \\ Geoecology“, 14476,Potsdam, Germany,oblustei@uni-potsdam.de,pustlauc@uni-potsdam.de \\ ${ }^{2}$ Aristotle University of Thessaloniki, Head of the School of Geology, 54006, Thessaloniki, Greece, \\ vavelidi@geo.auth.gr
}

\begin{abstract}
The main activity fields of the research group "Applied Geoecology" at the University of Potsdam are the development and tests of complex monitoring systems for water bodies and soils, together with the development of sustainable additives for vegetation restoration on ore mining dumps with extreme substratum parameters.

The dump substrata are translocated by surface waters and by aeolian processes to the surroundings affecting soil processes. A field spectrometer has been engineered, which can detect dam substrata in soils. Moreover, various soil additives were developed, enabling the establishment of vegetation on the extreme dump substrata. For all components there have been realised extensive tests in the framework of greenhouse and field experiments under different climate conditions on three continents, on various substrata and with varying plant species. All experiments were successful, even though no additional irrigation and no mineral fertilizer were allowed to be used, in order to realise the idea of a sustainable greening.

Keywords: Detection of allochthonous soil matter, soil improvement, re-greening, components of different soil additives.
\end{abstract}

\section{Introduction}

The main activity fields of the research group "Applied Geoecology", at the University of Potsdam are the development and tests of complex monitoring systems for water bodies, forestry and contaminated areas. Hereby, there is a close collaboration with international partners in Greece, Austria, China and South Africa.

For example, important hydrochemical data can be captured with the HYDROHERB-System for qanats and other water systems as well as with the complex monitoring system for gaseous and ionic water parameters.

Both systems have been developed for the industry market. With the help of them, it is possible to read out the data in situ, as well as transmit them to any computer in the world. All these systems can be programmed to capture the parameters automatically in a defined measurement interval (minutes to days). The data series can be efficiently processed further with Excel software. 


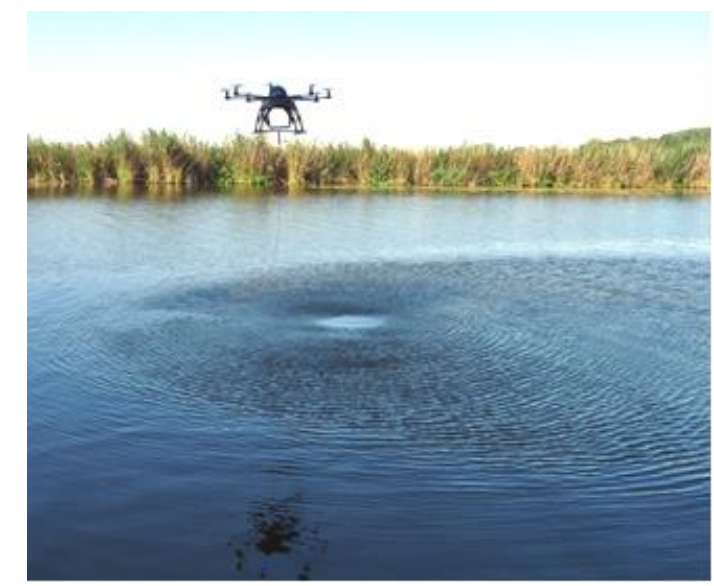

Figure 1 - Complex monitoring system for gaseous and ionic water parameters in action.

The computer-based data logging system for forestry owns an integrated soil chemical tool. With its assistance, the vegetations' growth characteristics can be detected. With this integrated soil chemical tool the most important soil parameters, such as $\mathrm{pH}$-value, soil moisture, conductivity and nitrate concentration can be captured quickly in situ. The tool can also be used separately. The data, in turn, can be transferred to any other computer.

However, one of the most important outcomes of the performed work is the development of sustainable additives for vegetation restoration on ore mining dumps with extreme substratum parameters. Why is it important?

These sites are characterised by

- extreme $\mathrm{pH}$-value,

- insufficient buffer capacity,

- deficiency of nutrients,

- too less bonded nutrients,

- absence of soil organic matter (SOM),

- extreme substratum density,

- extreme soil water conditions and instability of structures.

The ecological consequences shall be proven using the example of Rustenburg region (South Africa, North West Province), where the mining of PGMs and Chromium is dominating. The exploitation follows mostly in a depth of 1500 to $2000 \mathrm{~m}$ and is associated with a severe pollution of the environment. A major problem occurs with the disposal of the extracted ores that were grinded up and later washed in suspension on so called "tailings dams". Only in the Rustenburg area, there exist 14 landfills, which extents hit several square kilometres. On the tailings dams of platinum ore mining, the excavated materials' grain size is to be assigned to the silt fraction. Here, the $\mathrm{pH}$-value is in the alkaline range. Since there have only been extracted the PGMs, a considerable amount of heavy metals are still bound. Their contents exceed the internationally common boundary values many times over. Especially the chromium contents are extremely high (see fig. 2). 


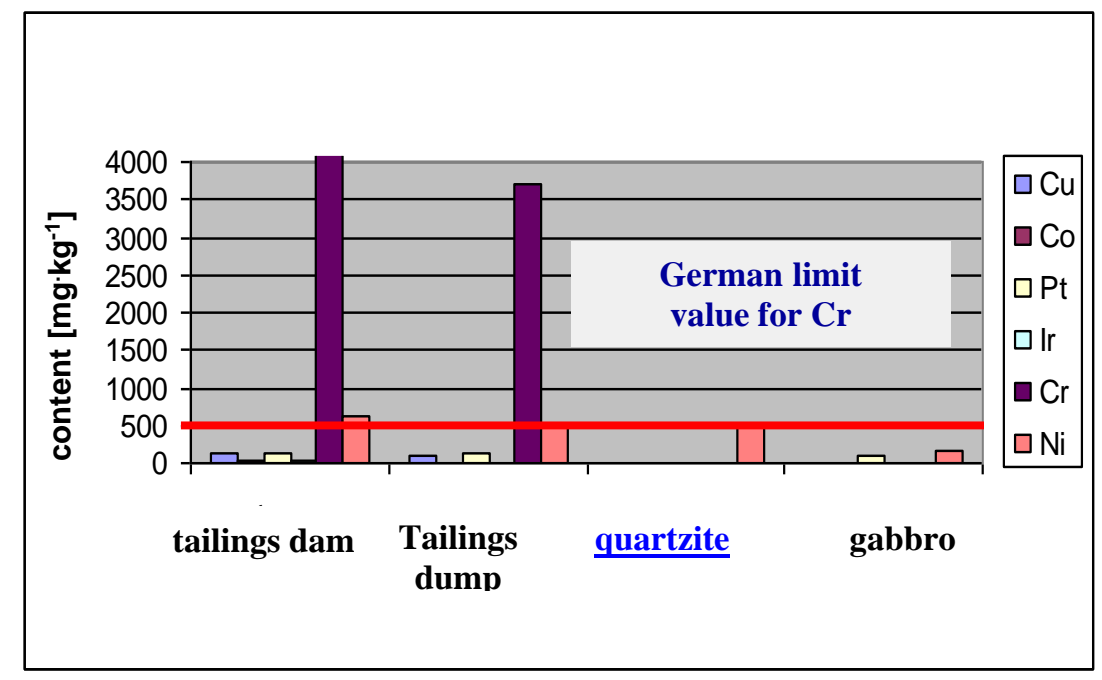

Figure 2 - Heavy metal contents of tailings substrata in comparison to regional genuine rocks.

\section{Results}

The fine substrate is shifted to the dams surroundings during rainy season by surface water and during dry season by aeolian processes. Those areas include settlements, water bodies and fields. In the farm soils there could be found substantial contents of platinum tailings substrate that is formed either diffusely or also as a layer (see fig. 3).

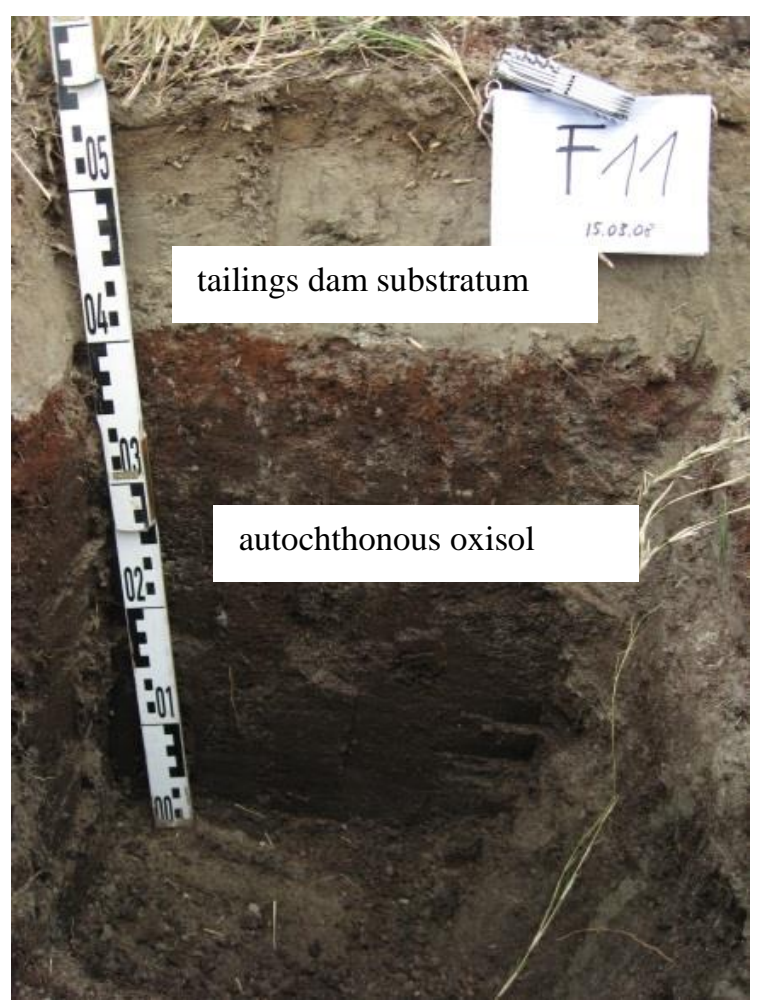

Figure 3 - Oxisol, overlaid by tailings dams subtrate (photo: S. Münzel). 
Those allochthonous substrates massively affect the soil developments. Their impacts had been a first investigational focus.

A second field of research resulted from the necessity of being able to detect those inputs of ore mining dumps substrata in the surrounding soils. After their deposition, they can no longer be distinguished by the naked eye from the substrata of vertisols and oxisols (see fig. 4).
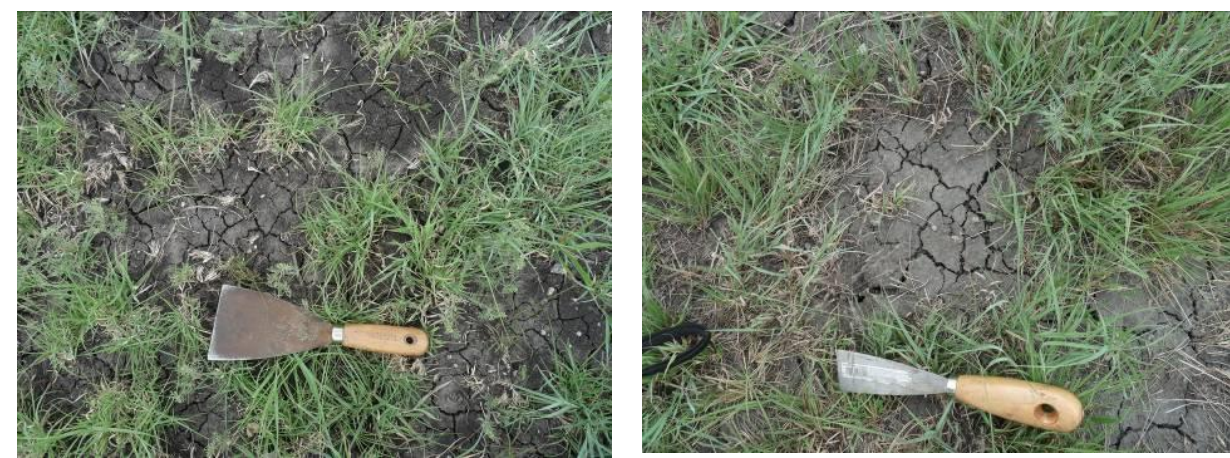

Figure 4 - Non contaminated vertisol (left), with platinum tailing dam substratum contaminated vertisol (right).

Therefore, a field spectrometer has been developed, which can detect substrata of gold tailings and platinum tailings in soils. At the test measurements in the Chalkidiki region, that served to the setup of the database, also partners from the Aristotle University Thessaloniki were involved. The user of this spectrometer (see fig. 5) can detect those substrata as well as organic compounds like mineral oils or paraffines in the soil.

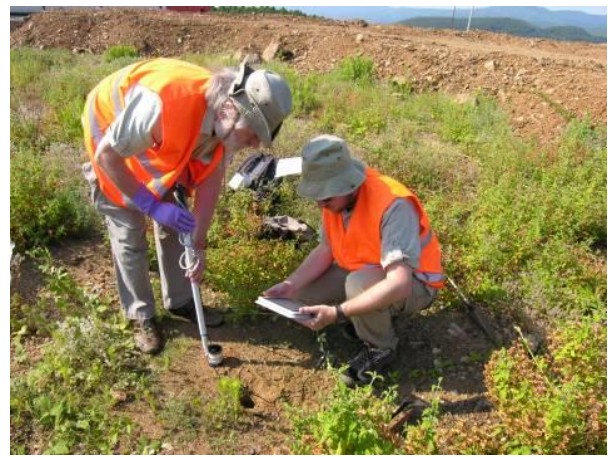

Figure 5 - Test measurements with the field spectrometer near Stratoni (Chalkidiki).

A further working direction was geared to reduce the intensity of tailings dump substrata shifting processes. Therefore sustainable soil additives have been developed, which can be mixed with the extreme dump substrata and thus enable the establishment of vegetation.

According to the diverse demands of soil and climate, five components (C1 - C5) aiming different effects have been developed. These effects are described briefly in figure 6 .

Moreover, a technique was developed, which consolidates the dump substrate by means of two solution components, so that its erosive shifting is substantially decreased.

For all components there have been realised extensive tests in the framework of greenhouse and field experiments under different climate conditions on three continents, on various substrata and with varying plant species. Certain examples are shown in figure 7. In any case the premise was that 
no additional irrigation and no mineral fertilizer were allowed to be used, in order to realise the idea of a sustainable greening.

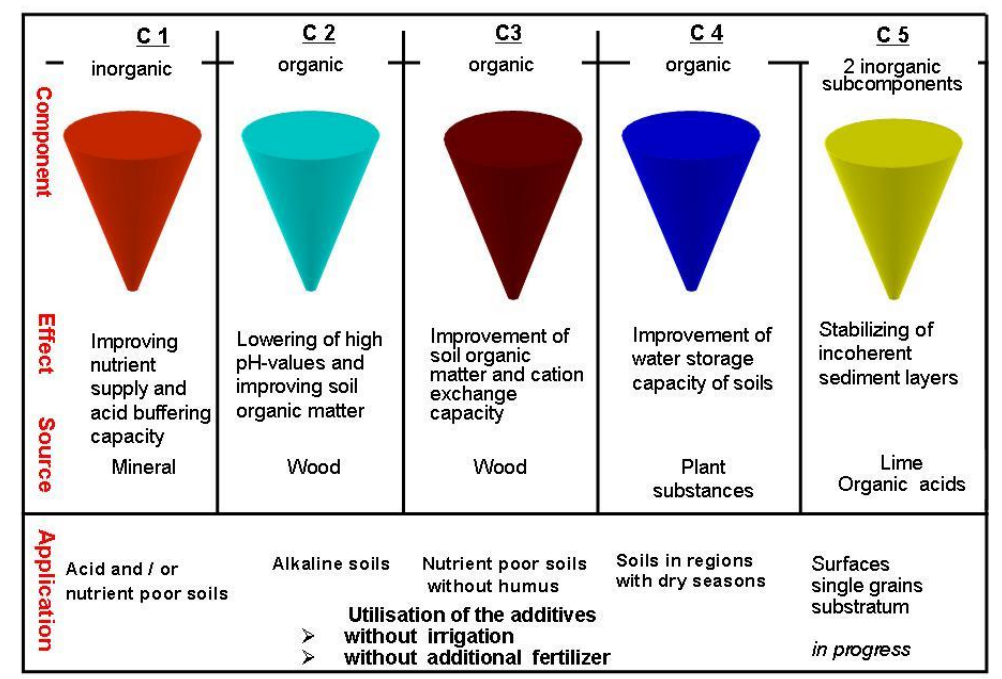

Figure 6 - Developed components (C1 - C5) of sustainable soil additives.

The aim was to evaluate biometric data like dry mass, growth rates and vitality (see for example fig. 8).

It became apparent, that one single component did not lead to an increase of biomass and an improvement of vitality in every case. The decisive factor was, that mixtures had to be com-pounded, which could then be optimised for individual substrate and climatic conditions.

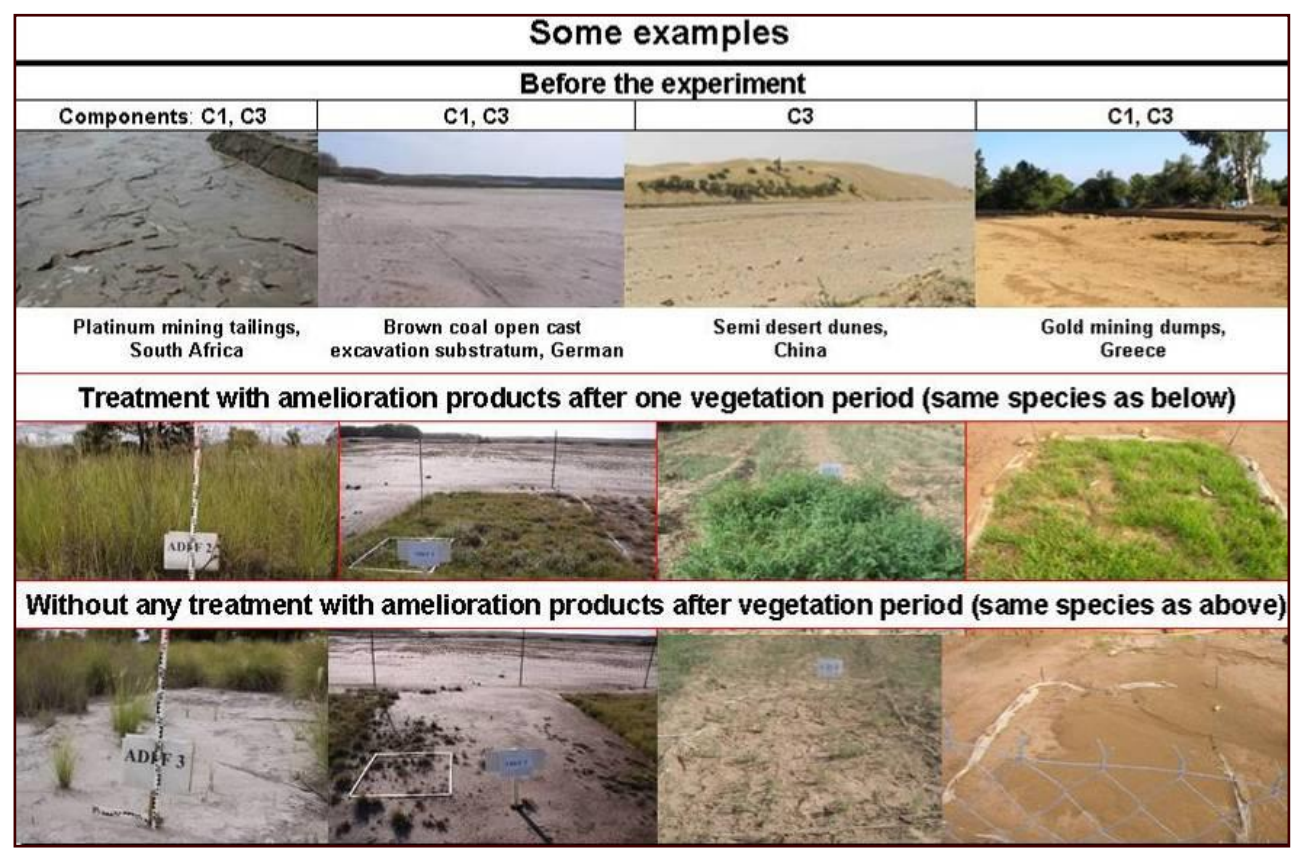

Figure 7 - Location of certain test sites and growth performances on various substrata. 
Dump substrata mostly show a small water storage capacity. This is why component C4 is important for a sustainable vegetation development, especially in areas with long dry periods. Even with a mixing in of about $1 \%$, an improvement of the water storage capacity in the soil occurs. The plants' drought stress can be decreased.

Results of the water retaining effect of component $\mathrm{C} 4$ are shown in figures 9 and 10 . When mixed in to low-nutrient sand, all plants were no longer irrigated after growing off. In combination with components $\mathrm{C} 1$ and $\mathrm{C} 3$ it became evident, that the plants that grew on substrata mixed with component $\mathrm{C} 4$, had produced way more dry mass (see fig. 9). Some individuals of Secale cereale have survived after four weeks without water addition. This shows, that a survival during a longer dry period is possible (fig. 10).

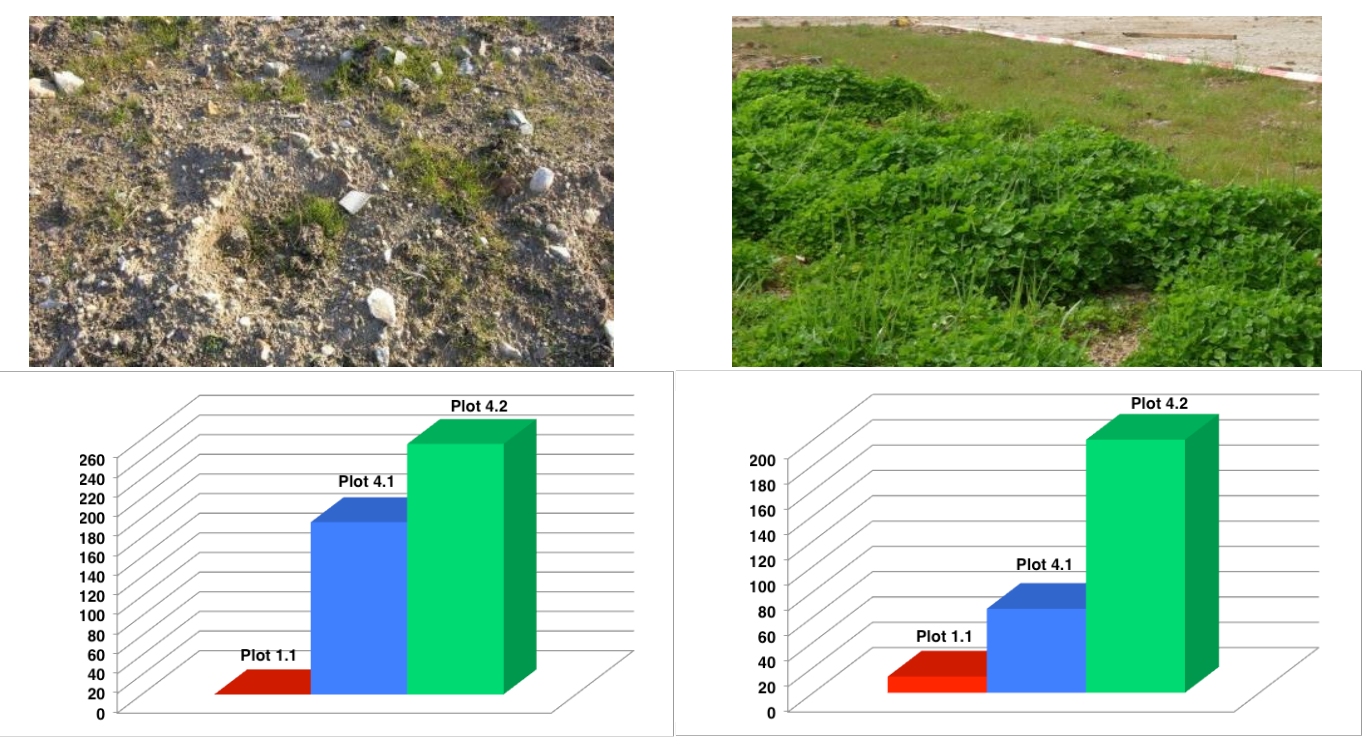

Figure 8 - Vegetation cover after one year development at the gold mining dump in Stratoni (Greece).

-above: without (plot 1.1, left) and with (plot 4.2, right) soil additives, but same species -below: subterranean (left) and over ground (right) dry matter of grass, plot 4.1 contents additives in lower percentage.

- above: without component $\mathrm{C} 4$

- below: with $1 \%$ component C4 


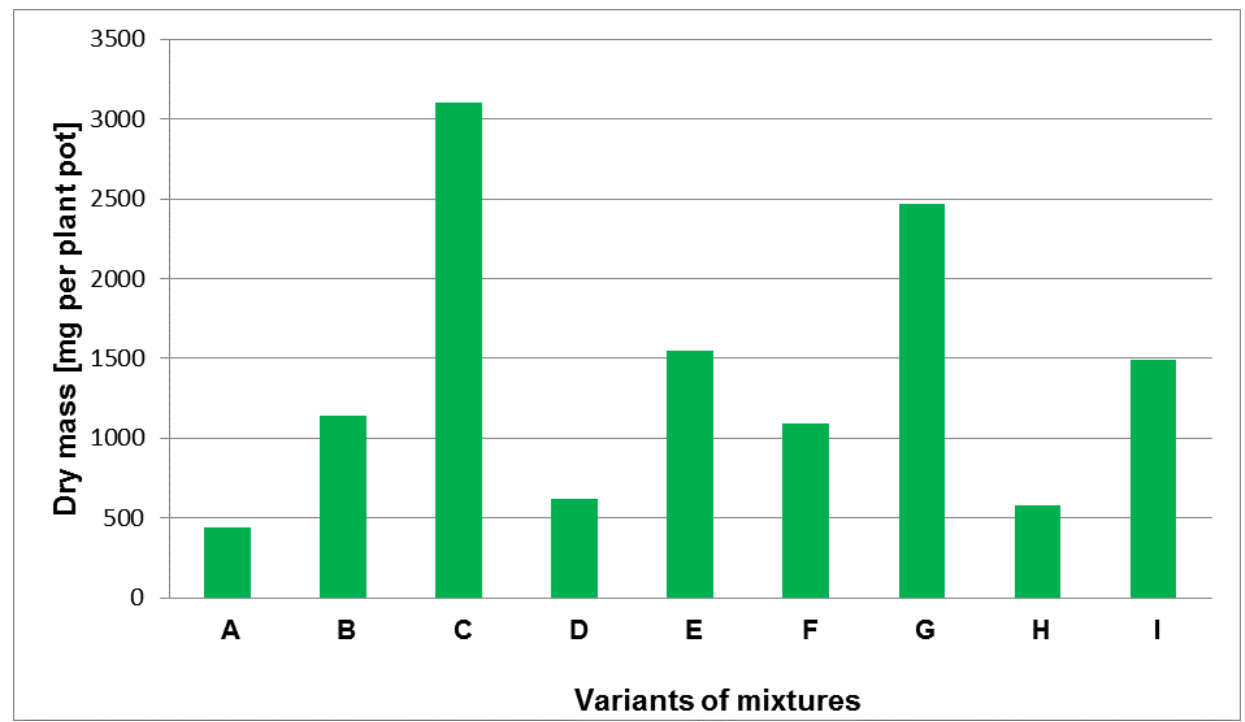

Figure 9 - Glasshouse experiment, average temperature $24^{\circ} \mathrm{C}$ : Overground dry mass of Secale cereale in plant pots with different contents of component $\mathrm{C} 4$ (trial $\mathrm{C}$ and $\mathrm{G} 1 \%$ content, trial $\mathrm{A}, \mathrm{D}$ and $\mathrm{H}$ without $\mathrm{C4}$ ) and components $\mathrm{C} 1$ and $\mathrm{C} 3$.
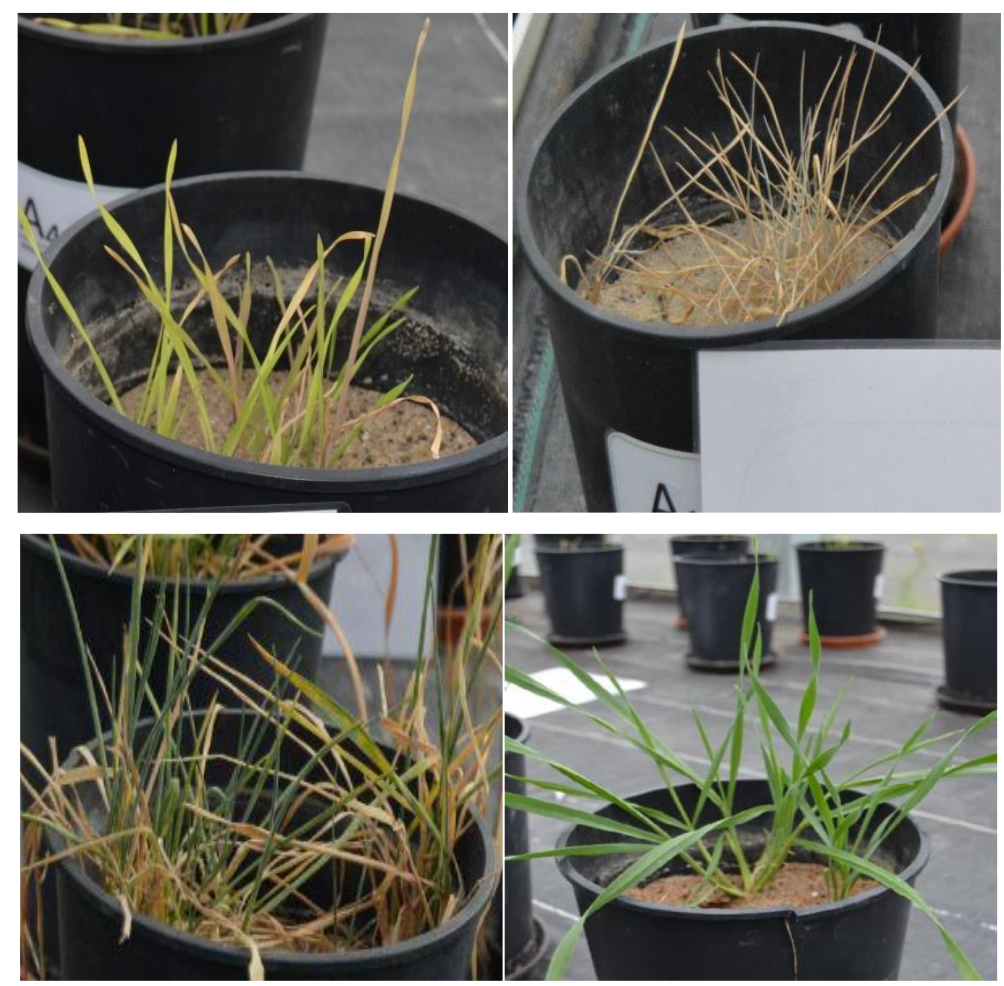

Figure 10 - Glasshouse experiment, average temperature $24^{\circ} \mathrm{C}$ : Secale cereale in plant pots by using different contents of component $\mathrm{C} 4$ after 14 days (left) and 28 days (right) without irrigation. 


\section{Summing up Discussion}

Substrata of ore mining dumps are often shifted to the dump surroundings by aeolian and fluvial processes. These foreign substrata massively influence the soil development, contaminants can attain to the food web.

Therefore a field spectrometer was developed, which can detect gold tailings and platinum tailings substrata in the soil.

The intensity of shifting processes can be decreased, if a vegetation is established on the dumps. For this reason, five components of sustainable soil additives, that can be mixed with the extreme dump substrata, have been developed.

For all components there have been realised extensive tests in the framework of greenhouse and field experiments under different climate conditions on three continents, on various substrata and with varying plant species. Since dump substrata mostly show a small water storage capacity, in regions with long dry periods, a water storing component is important for vegetation development. It could be shown that even with a mixing in of about $1 \%$, an improvement of water storage occurs in the soil. The drought stress of the plants can consequently be decreased.

\section{Acknowledgments}

An expression of thanks for scientific, technical or financial support is due to:

- Aristotle University of Thessaloniki (Greece), Laboratory of Forest Soil Science, Prof. Dimitris Alifragis

- Hellas Gold S.A., Stratoni (Greece), Ing. G. Bethlehem

- $\quad$ TOSOH Hellas A.I.C., Thessaloniki (Greece)

- Aquarius Platinum Lt. (South Africa), Ing. O. Faude

- North West University, Potchefstroom Campus, (South Africa), Unit Environmental Science and Management, Prof. L van Rensburg, P. van Deventer

- Beijing Forest University (China), College of Soil and Water Conservation, Prof. J. Guo

- Wurmitzer GmbH, Himmelberg (Austria)

- Universität Salzburg (Austria), Department of Geography and Geology, Ing. M. Marbach, Prof. H. Weingartner

- $\quad$ AIF Projekt GmbH, Berlin (Germany)

- UP Transfer GmbH, Potsdam (Germany)

- $\quad$ MLW intermed GmbH, Schöneiche (Germany)

- $\quad$ IPP Hydroconsult GmbH, Cottbus (Germany)

\section{References}

Since the research and development works are realised in the framework of industry projects, references only exist in the form of unpublished reports. 